\title{
Universal short-distance structure of the single-particle spectral function of dilute Fermi gases
}

\author{
William Schneider and Mohit Randeria \\ Department of Physics, The Ohio State University, Columbus, Ohio 43210, USA
}

(Received 15 October 2009; published 3 February 2010)

\begin{abstract}
We show that the universal $1 / k^{4}$ tail in the momentum distribution of dilute Fermi gases implies that the spectral function $A(\mathbf{k}, \omega)$ must have weight below the chemical potential for large momentum $k \gg k_{F}$, with observable consequences in radio-frequency spectroscopy experiments. We find that this incoherent spectral weight is centered around $\omega \simeq-\epsilon(\mathbf{k})$ in a range of energies of order $v_{F} k$. This "bending back" in the dispersion, while natural for superfluids, is quite surprising for normal gases. This universal structure is present in the hard-sphere gas as well as in the Fermi-liquid ground state of the highly imbalanced, attractive gas near unitarity. We argue that, even in the BCS superfluid, this bending back at large $k$ is dominated by interaction effects which do not reflect the pairing gap.
\end{abstract}

DOI: 10.1103/PhysRevA.81.021601

PACS number(s): 03.75.Ss, 67.85.-d, 32.30.Bv

The spectral function $A(\mathbf{k}, \omega)=-\operatorname{Im} G\left(\mathbf{k}, \omega+i 0^{+}\right) / \pi$ of the single-particle Green's function $G$ is of fundamental interest in many-body physics [1,2]. In addition to information about the spectrum and dynamics of single-particle excitations, it is also directly related to thermodynamic functions of a many-particle system. Very recently there has been experimental progress in measuring (the occupied part of) $A(\mathbf{k}, \omega)$ in strongly interacting Fermi gases [3,4], using a momentumresolved version [5] of radio-frequency (rf) spectroscopy [6-8]. These measurements [5] of $A(\mathbf{k}, \omega)$ for ultracold atomic gases are the analog of angle-resolved photoemission, which has given deep insights into novel materials.

In this article we uncover the remarkable universal large- $k$ structure of $A(\mathbf{k}, \omega)$ for dilute gases with observable effects in rf experiments. Our investigation was motivated by the elucidation of the universal ultraviolet structure of equal-time correlations by Tan and others $[9,10]$. One of his central results is the universal $k \gg k_{F}$ behavior of the momentum distribution $n_{\sigma}(\mathbf{k}) \simeq C / k^{4}$, where $C$ is the "contact" $[9,10]$. Using the $T=$ 0 sum rule $\int_{-\infty}^{0} d \omega A(\mathbf{k}, \omega)=n(\mathbf{k})$, this necessarily implies that $A(\mathbf{k}, \omega)$ has weight below the chemical potential $(\omega<$ $0)$ for $k \gg k_{F}$. This is "incoherent" spectral weight, not associated with the coherent Landau quasiparticle.

We ask the question: Where is this incoherent spectral weight located for $k \gg k_{F}$ ? The surprising answer is that the incoherent part of the $\omega$ versus $k$ dispersion goes like $-\epsilon(\mathbf{k})=-k^{2} / 2 m$, "bending back" away from the chemical potential at large $k$. While this is expected in BCS theory and its generalizations for a paired superfluid, we argue that this unusual dispersion is a universal feature of all dilute Fermi gases, even those with a normal (non-superfluid) ground state. We find that the spectral weight of $C / k^{4}$ in $A(\mathbf{k}, \omega)$ is centered around $\omega \simeq-\epsilon(\mathbf{k})$ in a range of energies of order $v_{F} k$ for normal Fermi gases. Most of the spectral weight $\left(1-C / k^{4}\right)$ is, of course, centered around $\omega \approx+\epsilon(\mathbf{k})$, but these states are not occupied and do not contribute to $n(\mathbf{k})$.

This bending back is clearly visible in the data of Ref. [5] for attractive fermions near unitarity and near or above $T_{c}$. However, it is hard to separate the effects of the finite temperature pairing pseudogap [11] and normal state interaction effects. In particular, a bending back of the dispersion above $T_{c}$ cannot by itself be used as evidence for a pairing pseudogap in view of the normal state results described below.
We first focus on two systems where the ground state is a normal Fermi liquid: (a) the hard-sphere dilute Fermi gas and (b) the highly imbalanced attractive Fermi gas. We then turn to the superfluid ground state, where we argue that, in the BCS limit, the unusual dispersion is dominated by interaction effects rather than the effect of pairing. We conclude with implications for $\mathrm{rf}$ spectroscopy experiments.

Dilute repulsive Fermi gas. We begin with the threedimensional hard-sphere Fermi gas with dispersion $\epsilon(\mathbf{k})=$ $k^{2} / 2 m$, mass $m$, density $n=k_{F}^{3} / 3 \pi^{2}$, and scattering length $a>0$ with $n a^{3} \ll 1$. (We set $\hbar=k_{B}=1$.) Its thermodynamic and Fermi-liquid properties were studied by Galitskii and Lee, Yang, and Huang; see Sec. 5 of Ref. [1]. The high- $k$ tail was also calculated [12,13]: $n(\mathbf{k}) \simeq\left(k_{F} a\right)^{2}(2 / 3 \pi)^{2}\left(k_{F} / k\right)^{4}$. Here we compute $A(\mathbf{k}, \omega)$.

In the low-density limit $n a^{3} \ll 1$, the most important physical process is repeated scattering in the particleparticle channel. The corresponding sum of ladder diagrams $\Gamma$ is given by $\Gamma^{-1}(Q)=1 / g-L(Q)$, where $Q=$ $\left(\mathbf{Q}, i Q_{\ell}\right)$ with $i Q_{\ell}=i 2 \ell \pi T$ and the bare interaction $g$ is related to $a$ via $1 / g=m /(4 \pi a)-\sum_{\mathbf{k}} 1 /[2 \epsilon(\mathbf{k})]$. Further $L(Q)=T \sum_{k} G^{0}(k+Q) G^{0}(-k)$ where $k=\left(\mathbf{k}, i k_{n}\right)$ with $i k_{n}=i(2 n+1) \pi T$ and $G^{0}(k)=1 /\left[i k_{n}-\xi(\mathbf{k})\right]$ is the bare Green's function with the energy $\xi(\mathbf{k})=\epsilon(\mathbf{k})-\mu$ measured with respect to the chemical potential $\mu$ [14]. Note that one can obtain an analytically closed form expression [15] for $L\left(\mathbf{Q}, \Omega+i 0^{+}\right)$. For the hard-sphere gas we can make a further simplification, $\Gamma \approx g+g^{2} L$.

The Matsubara self-energy $\Sigma(k)=T \sum_{q} \Gamma(k+q) G^{0}(q)$ yields $\Sigma\left(\mathbf{k}, i k_{n} \rightarrow \omega+i 0^{+}\right)=\operatorname{Re} \Sigma+i \operatorname{Im} \Sigma$, where

$$
\operatorname{Im} \Sigma(\mathbf{k}, \omega)=\sum_{\mathbf{q}} \operatorname{Im} \Gamma(\mathbf{Q}, \Omega)\{\Theta[-\xi(\mathbf{q})]-\Theta(-\Omega)\}
$$

at $T=0$, with $\mathbf{Q}=\mathbf{k}+\mathbf{q}$ and $\Omega=\omega+\xi(\mathbf{q}) \cdot \operatorname{Re} \Sigma$ is obtained numerically by a Kramers-Kronig transform [16] on $\operatorname{Im} \Sigma$. The spectral function is then obtained using $A(\mathbf{k}, \omega)=-\operatorname{Im}[\omega-$ $\xi(\mathbf{k})-\Sigma(\mathbf{k}, \omega)]^{-1} / \pi$ and plotted in Fig. 1 on a log scale. We see that the most intense feature, corresponding to the Landau quasiparticle near $k_{F}$, tracks $\omega \approx+\xi(\mathbf{k})$, up to many-body renormalizations [17]. However our main interest is in the much less intense, incoherent spectral feature that follows an $\omega=-\epsilon(\mathbf{k})$ dispersion and dominates $n(\mathbf{k})$ at large $k$. 


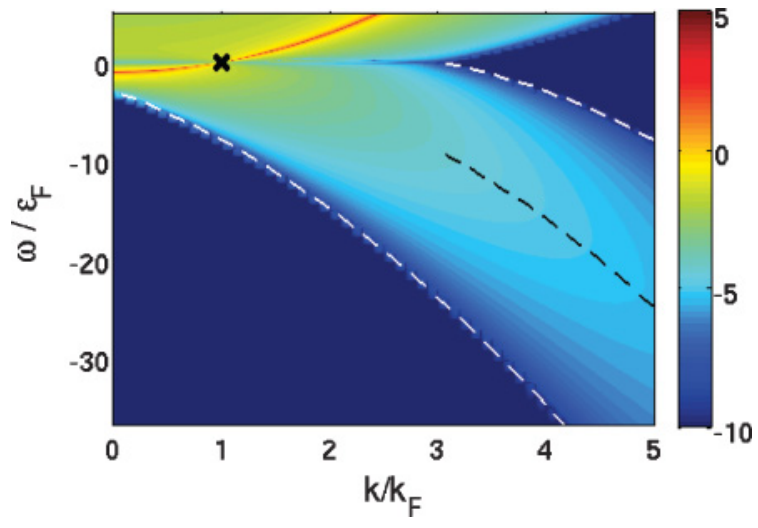

FIG. 1. (Color online) Logarithmic intensity plot of $A(\mathbf{k}, \omega) \epsilon_{F} /\left(k_{F} a\right)^{2}$ for the repulsive Fermi gas $\left(k_{F} a=0.1\right.$, $n a^{3}=3.4 \times 10^{-5}$ ). The most intense (red) line at $\omega \approx \xi_{k}$ is the quasiparticle. We focus on the unusual dispersion centered around $\omega=-\epsilon(\mathbf{k})$ (black dashed line) in the range $\omega=-\epsilon(\mathbf{k})-3 \epsilon_{F} \pm$ $2 v_{F} k$ (white dashed lines); see text.

To understand this bending back, we write $A \approx$ $|\operatorname{Im} \Sigma(\mathbf{k}, \omega)| /\left\{\pi[\omega-\epsilon(\mathbf{k})]^{2}\right\}$. We need to determine when $\operatorname{Im} \Sigma(k, \omega)$ is nonzero for $k \gg k_{F}$ and $\omega<0$. To understand our result qualitatively, consider the diagram in Fig. 2. The dominant contribution comes from small values of both $|\mathbf{Q}|$ and $\Omega$. (For large values of these variables there is no spectral weight $\operatorname{Im} \Gamma$ for two-particle scattering.) Thus $\mathbf{q} \simeq-\mathbf{k}$ and $\omega \simeq-\xi(\mathbf{q}) \simeq-\epsilon(\mathbf{k})$ for $k \gg k_{F}$. This shows that $A \neq 0$ for $\omega$ around negative $\epsilon(\mathbf{k})$.

To make this more quantitative, we use Eq. (1). From the structure of $L(\mathbf{Q}, \Omega)$, it follows that $\operatorname{Im} \Gamma(\mathbf{Q}, \Omega) \neq$ 0 when $\Omega \geqslant \Omega_{0}(Q) \equiv \min _{\mathbf{p}}[\xi(\mathbf{p}+\mathbf{Q} / 2)+\xi(-\mathbf{p}+\mathbf{Q} / 2)]=$ $\epsilon(Q) / 2-2 \mu$; see Fig. 2. From the difference of $\Theta$ functions, $k_{F} \leqslant q \leqslant q_{\max }(\omega) \equiv k_{F}\left(1+|\omega| / \epsilon_{F}\right)^{1 / 2}$. This implies that $-|\omega| \leqslant \Omega \leqslant 0$. Together with the kinematical constraint $|k-q| \leqslant Q \leqslant k+q$, this leads to $Q_{\min }=\mid k-$ $q_{\text {max }}(\omega) \mid$. For nonzero $\operatorname{Im} \Sigma$ we thus need the kinematically allowed region (shaded rectangle in Fig. 2) to overlap with $\Omega \geqslant \Omega_{0}(Q)$. This leads to the simple condition $Q_{\min } \leqslant Q_{0}$, where the definition $\Omega\left(Q_{0}\right)=0$ leads to $Q_{0}=2 k_{F}$. (We have

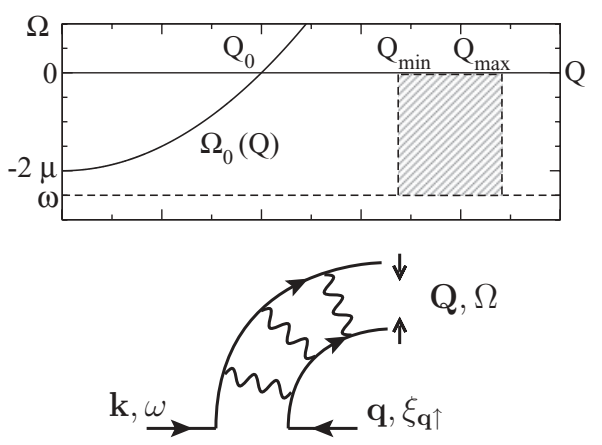

FIG. 2. Top: Kinematics of the processes that contribute to imaginary self-energy in Eq. (1). $\operatorname{Im} \Sigma$ is nonzero when the shaded rectangle (allowed by kinematics and thermal factors) overlaps with the region $\Omega>\Omega_{0}(Q)$ (in which $\operatorname{Im} \Gamma$ is nonzero). This leads to the condition $Q_{\min } \leqslant Q_{0}$. Bottom: Diagram contributing to $\operatorname{Im} \Sigma$.

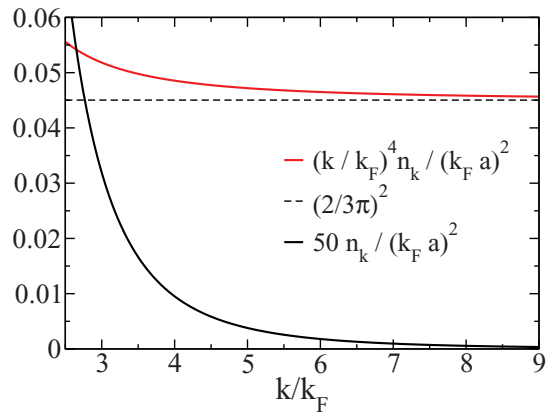

FIG. 3. (Color online) Momentum distribution tail for the dilute repulsive Fermi gas with $n a^{3}=3.4 \times 10^{-5}$.

also found, but do not discuss here, the $\omega>0$ threshold for $A \neq 0$.)

Solving $\left|k-q_{\max }(\omega)\right|=2 k_{F}$, we find $A(\mathbf{k}, \omega<0) \neq 0$ in the range of energies $\omega=-\epsilon(\mathbf{k})-3 \epsilon_{F} \pm 2 v_{F} k$; see Fig. 1 . For $k \gg k_{F}$, this simplifies to $|\omega+\epsilon(\mathbf{k})| \leqslant 2 v_{F} k$. Although the width of this range grows linearly with $k$, it becomes small relative to the central energy which grows like $-k^{2}$ for large $k$. We plot in Fig. 3 the $n(\mathbf{k})$ tail using $\int_{-\infty}^{0} d \omega A(\mathbf{k}, \omega)$ and find that it agrees with the analytical result [12]. The incoherent spectral weight in $A\left(k \gg k_{F}, \omega\right)$ in the interval $|\omega+\epsilon(\mathbf{k})| \leqslant$ $2 v_{F} k$ is thus precisely $\left(2 k_{F} a / 3 \pi\right)^{2}\left(k_{F} / k\right)^{4}$.

Highly imbalanced Fermi gas. We next turn to a twocomponent attractive Fermi gas with scattering length $a$ tuned through a broad Feshbach resonance [18]. While the ground state for equal spin populations is a superfluid exhibiting the BCS-BEC crossover, we consider the different regime of large spin imbalance $n_{\uparrow} / n_{\downarrow}$. There is by now considerable theoretical $[19,20]$ and experimental evidence [7,8] that, for a sufficiently large imbalance, superfluidity is destroyed for a large range of values of $a$, including unitarity $|a|=\infty$, and the ground state is a (partially polarized) normal Landau Fermi liquid.

For large $|a|$, we use the number of fermion species $2 \mathcal{N}$ with an $\operatorname{Sp}(2 \mathcal{N})$-invariant interaction as an artificial parameter to control the calculation in a large- $\mathcal{N}$ expansion $[15,20]$. To first order in $1 / \mathcal{N}$, ladder diagrams in the $p$ - $p$ channel determine the self-energy. The resulting expressions are similar to those used above and we show them schematically, highlighting the differences that arise from spin imbalance. We now have $L=T \sum G_{\uparrow}^{0} G_{\downarrow}^{0}$ where $G_{\sigma}^{0}(k)=1 /\left[i k_{n}-\xi_{\sigma}(\mathbf{k})\right]$ with $\xi_{\sigma}(\mathbf{k})=\epsilon(\mathbf{k})-\mu_{\sigma}$. The minority self-energy is given by $\Sigma_{\downarrow}=T \sum \Gamma G_{\uparrow}^{0} . \operatorname{Im} \Sigma_{\downarrow}$ is then given by Eq. (1) with $\xi$ replaced by $\xi_{\uparrow}$ both in the $\Theta$ function and in the definition of $\Omega$.

We can analytically determine the energy range for which $\operatorname{Im} \Sigma$, and hence $A$, is nonzero. In Fig. 2 we must now use $\Omega_{0}(Q) \equiv \epsilon(Q) / 2-2 \mu$ with $2 \mu=\mu_{\uparrow}+\mu_{\downarrow}$. The final result [21] is that, for $k \gg k_{F}$ and $\omega<0, A(\mathbf{k}, \omega)$ can be nonzero only in the range of energies $|\omega+\epsilon(\mathbf{k})| \leqslant \alpha v_{F \uparrow} k$ where $\alpha=\sqrt{2\left(1+\epsilon_{F \downarrow} / \epsilon_{F \uparrow}\right)}$.

For concreteness, we focus here on unitarity $|a|=\infty$. $A(\mathbf{k}, \omega)$ for the highly imbalanced $\left(n_{\downarrow} / n_{\uparrow}=0.01\right)$ unitary gas is shown in Fig. 4. We have also verified that we get a $1 / k^{4}$ tail for $n(\mathbf{k})$ in this system. Our calculation of $A(\mathbf{k}, \omega)$ is controlled only within the $1 / \mathcal{N}$ expansion. Note, however, 


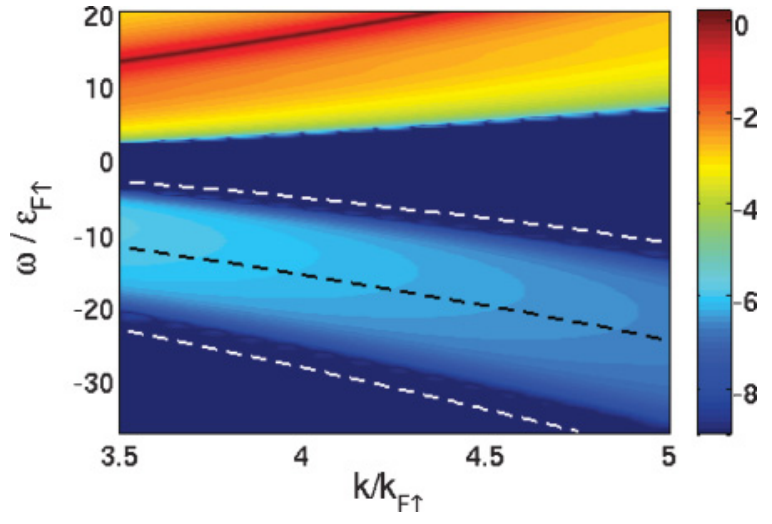

FIG. 4. (Color online) Logarithm intensity plot of $A_{k}(\omega) \epsilon_{F \uparrow}$ for minority particles in the unitary Fermi gas with imbalance $n_{\downarrow} / n_{\uparrow}=$ 0.01 . The white dashed lines $\omega=-\epsilon(\mathbf{k}) \pm \alpha v_{F \uparrow} k$ are derived in the text; the black dashed line is $\omega=-\epsilon(\mathbf{k})$.

that the singularity structure in the large- $k$ limit is determined only by short-distance properties of the two-body problem in vacuum, while the strength of the singularity $C$ depends on the many-body state. The ladder approximation is exact for the two-body problem. Thus we expect the bending back in the spectral function to be robust beyond the $1 / \mathcal{N}$ expansion.

Superfluid state. We now turn to a discussion of the superfluid ground state for a system with equal densities of up and down spins and an interaction described by scattering length $a$. Unlike the normal Fermi liquids described above, a branch of the dispersion that tracks $-\epsilon_{\mathbf{k}}$ at large $k$ is very natural for the fermionic excitations in a superfluid [22]. Nevertheless, even in this case, our analysis gives important quantitative insights.

In BCS mean-field theory the spectral function $A_{\mathrm{MF}}(\mathbf{k}, \omega)=v_{\mathbf{k}}^{2} \delta[\omega+E(\mathbf{k})]+u_{\mathbf{k}}^{2} \delta[\omega-E(\mathbf{k})]$ where $v_{\mathbf{k}}^{2}=$ $1-u_{\mathbf{k}}^{2}=[1-\xi(\mathbf{k}) / E(\mathbf{k})] / 2$. The excitation energy $E(\mathbf{k})=$ $\sqrt{\xi^{2}(\mathbf{k})+\Delta^{2}}$ with $\Delta$ being the energy gap. For $k \gg k_{F}$, $E(\mathbf{k}) \approx \epsilon(\mathbf{k})$ and $v_{\mathbf{k}}^{2} \approx \Delta^{2} / 2 \epsilon^{2}(\mathbf{k})$, so that $A_{\mathrm{MF}}\left(k \gg k_{F}, \omega<\right.$ $0) \approx\left[\Delta^{2} / 2 \epsilon^{2}(\mathbf{k})\right] \delta[\omega+\epsilon(\mathbf{k})]$. Thus we see that particle-hole mixing in the superfluid ground state naturally leads to a bending back of the dispersion.

However, there is a (large) quantitative problem with this result even in the BCS limit $\left(1 / k_{F} a \ll-1\right)$, where one might have expected it to be the most accurate. Using $n(\mathbf{k})=\int_{-\infty}^{0} d \omega A(\mathbf{k}, \omega)$, or directly from BCS theory, one finds that the momentum distribution $n_{\mathrm{MF}}(\mathbf{k})=v_{\mathbf{k}}^{2} \approx \Delta^{2} / 2 \epsilon^{2}(\mathbf{k})=$ $C_{\mathrm{MF}} / k^{4}$ for $k \gg k_{F}$. The problem is that the contact estimated from BCS theory, $C_{\mathrm{MF}} \sim \Delta^{2} \sim \exp \left(-1 / k_{F}|a|\right)$, is exponentially small in $|a|$. However, the exact answer $[9,10]$ in the BCS limit is $C=4 \pi^{2} n^{2} a^{2}$ as $a \rightarrow 0^{-}$. To understand why BCS theory gets the wrong answer for $C$ we use the adiabatic relation [9] $d \mathcal{E} / d a=\hbar^{2} C /\left(4 \pi m a^{2}\right)$. As shown in Ref. [23], interaction effects lead to power-law corrections in $|a|$ in the ground-state energy density $\mathcal{E}$, which are numerically much more important than the essentially singular corrections coming from pairing. In the extreme BCS limit, the contact is dominated by the Hartree term in $\mathcal{E}$ with calculable corrections [23].
Thus the actual $A\left(k \gg k_{F}, \omega<0\right)$, even in the BCS limit, is dominated by interaction effects beyond BCS mean-field theory. This results in the spectral weight $C \sim|a|^{2}$ arising from interaction effects which exist even in the normal state, rather than resulting from pairing, which only makes an exponentially small contribution.

Implications for rf spectroscopy. The physical effects we have discussed above lead to directly observable consequences in $\mathrm{rf}$ spectroscopy experiments where an $\mathrm{rf}$ pulse is used to transfer atoms from one hyperfine level to another. The interpretation of these experiments is often complicated by two difficulties: the inhomogeneity of trapped gases and severe final state interactions. The first problem has been solved in the usual ("angle-integrated") rf experiments using tomographic techniques. Final state effects are not an issue in ${ }^{40} \mathrm{~K}$ [5] and have been eliminated in ${ }^{6} \mathrm{Li}$ by suitable choices of hyperfine levels [7,8]. We emphasize that but for this it would be very difficult to disentangle strong interactions in the many-body state (self-energy effects) from final state effects (vertex corrections) [24]. We thus work in the (now experimentally relevant) limit where we ignore all final state interactions.

Linear response theory then leads to the $\mathrm{rf}$ absorption intensity $I_{\sigma}(\mathbf{k}, \omega)=A_{\sigma}\left[\mathbf{k}, \xi_{\sigma}(\mathbf{k})-\omega\right] f\left[\xi_{\sigma}(\mathbf{k})-\omega\right]$ where $\omega$ is the rf shift. The Fermi function $f(\omega)$ ensures that only occupied states can be excited by the probe. We set the multiplicative factor of the rf matrix element to unity so that $\int d \omega \sum_{\mathbf{k}} I_{\sigma}(\mathbf{k}, \omega)=N_{\sigma}$.

Angle-resolved rf experiments [5] directly probe (the occupied part of) the spectral function $A(\mathbf{k}, \omega)$ and can see its unusual dispersion for $k \gg k_{F}, \omega<0$. As already noted at the beginning of this article, one cannot identify this bending back with pairing pseudogap physics [11] since this universal feature also occurs in normal Fermi liquids.

The consequences of our results for the angle-averaged rf experiments, which measure $I_{\sigma}(\omega)=\sum_{\mathbf{k}} I_{\sigma}(\mathbf{k}, \omega)$, are more subtle. We now show that the unusual dispersion at large $\mathbf{k}$ has a quantitative effect on the prefactor of the universal high- $\omega$ tail [25] in $I_{\sigma}$. We rewrite $I_{\sigma}(\omega)=$ $\sum_{\mathbf{k}} \int_{-\infty}^{0} d \Omega A_{\sigma}(\mathbf{k}, \Omega) \delta\left[\Omega-\xi_{\sigma}(\mathbf{k})+\omega\right]$ at $T=0$. In the $\omega \rightarrow$ $\infty$ limit, large negative $\Omega$ values, centered about $\Omega \approx-\epsilon(\mathbf{k})$, dominate. We thus find $I_{\sigma}(\omega \rightarrow \infty) \simeq \sum_{\mathbf{k}} n_{\sigma}(\mathbf{k}) \delta\left(\omega-2 \epsilon_{\mathbf{k}}\right)$. Using $n_{\sigma}(\mathbf{k}) \approx C / k^{4}$ for $k \gg k_{F}$ [9] we thus find that $I_{\sigma}(\omega \rightarrow$ $\infty) \approx\left(C / 4 \pi^{2} \sqrt{m}\right) \omega^{-3 / 2}$ [26]. The characteristic power law is independent of the phase (normal or superfluid) of the Fermi gas, though the value of $C$ does depend on the many-body state.

The $\omega^{-3 / 2}$ tail has been discussed by various authors [22,25,27]. First, we have derived this result analytically under very general conditions [26]. Second, our results show that one must be very careful in interpreting its origin. We emphasize that this tail and the bending back of the dispersion, to which it is intimately related, arise from short-distance "contact" physics and should not by themselves be taken as evidence for pairing.

Conclusions. We have shown that there is an unusual feature in the large-momentum structure of the single-particle spectral function of all dilute Fermi gases, normal or superfluid, which is closely related to the universal short-distance features discussed by Tan and others [9,10]. This is an incoherent branch of the dispersion, where $\omega$ goes like negative $\epsilon_{\mathbf{k}}$ [28], 
that is quite unexpected in a normal Fermi gas. Nevertheless, this is exactly what we find in the two systems where the ground state is known to be a normal Landau Fermi liquid: the hard-sphere Fermi gas and the highly imbalanced, attractive Fermi gas. Even in a BCS superfluid, we show that this bending back at large $k$ is dominated by interaction effects rather than by the pairing gap.

Note Added. Recently, we learned of a work by Combescot, Alzetto, and Leyronas [28], where the approximation
$\operatorname{Im} \Sigma\left(k \gg k_{F}, \omega<0\right) \propto \delta(\omega+\epsilon(\mathbf{k}))$ is used which leads to a sharp feature in $A(\mathbf{k}, \omega)$. While this may be sufficient for computing "integrated" quantities like $n(\mathbf{k})$, it does not capture the incoherent structure in $A(\mathbf{k}, \omega)$ described here.

We acknowledge discussions with D. Jin and E. Taylor and support from NSF-DMR 0706203 and ARO W911NF-08-10338 .
[1] A. A. Abrikosov, L. P. Gorkov, and I. Dzyaloshinski, Methods of Quantum Field Theory in Statistical Physics (Dover, New York, 1963).

[2] L. Kadanoff and G. Baym, Quantum Statistical Mechanics (Benjamin, Elmsford, NY, 1962).

[3] I. Bloch, J. Dalibard, and W. Zwerger, Rev. Mod. Phys. 80, 885 (2008).

[4] S. Giorgini, L. P. Pitaevskii, and S. Stringari, Rev. Mod. Phys. 80, 1215 (2008).

[5] J. T. Stewart, J. P. Gaebler, and D. S. Jin, Nature (London) 454, 744 (2008); D. S. Jin (private communication).

[6] C. Chin et al., Science 305, 1128 (2004).

[7] A. Schirotzek, Y. I. Shin, C. H. Schunck, and W. Ketterle, Phys. Rev. Lett. 101, 140403 (2008).

[8] A. Schirotzek, C. H. Wu, A. Sommer, and M. W. Zwierlein, Phys. Rev. Lett. 102, 230402 (2009).

[9] S. Tan, Ann. Phys. 323, 2952 (2008); 323, 2971 (2008); 323, 2987 (2008).

[10] E. Braaten and L. Platter, Phys. Rev. Lett. 100, 205301 (2008); F. Werner, L. Tarruell, and Y. Castin, Eur. Phys. J. B 68, 401 (2009); S. Zhang and A. J. Leggett, Phys. Rev. A 79, 023601 (2009).

[11] M. Randeria, in Proceedings of the International School of Physics "Enrico Fermi," Course CXXXVI on High Temperature Superconductivity, edited by G. Iadonisi, J. R. Schrieffer, and M. L. Chiafalo (IOS Press, Amsterdam, 1998); e-print arXiv:cond-mat/9710223.

[12] V. A. Belyakov, Sov. Phys. JETP 13, 850 (1961). Note that Ref. [1] has a typographical error in the last equation of (5.24): the prefactor of $k^{-4}$ is too large by a factor of 4 .

[13] For a hard-sphere gas, $a=r_{0}$, the range, and the "large"- $k$ regime where $n(\mathbf{k}) \sim k^{-4}$ is $k_{F} \ll k \ll 1 / r_{0}$. Thus, unlike Ref. [9], where $a \gg r_{0}$, we cannot set $r_{0}=0$.

[14] We use $\mu=\epsilon_{F}$ in $L$ and $G^{0}$ since the corrections to $\mu$ are of order $k_{F} a$. This is also done for the large- $\mathcal{N}$ calculation for the polarized Fermi gas below.

[15] P. Nikolic and S. Sachdev, Phys. Rev. A 75, 033608 (2007).

[16] The simplification $\Gamma \approx g+g^{2} L$ for the hard sphere gas is valid only for $\omega \ll 1 / m a^{2}$. $\operatorname{Im} \Sigma$ grows indefinitely for larger $\omega$. We subtract out this singular behavior $S(z)=$ $-i(2 \sqrt{2} / 3 \pi)\left(k_{F} a\right)^{2} \sqrt{\epsilon_{F} z}$ and then the Kramers-Kronig transform $\operatorname{Im} \widetilde{\Sigma}=\operatorname{Im} \Sigma-\operatorname{Im} S$. The real self-energy is then $\operatorname{Re} \Sigma=$ $\operatorname{Re} \tilde{\Sigma}+\operatorname{Re} S$

[17] We have checked our numerics against known results [1] for chemical potential $\mu$, quasiparticle residue $Z$, effective mass $m^{*}$, and the scattering rate near the Fermi surface.

[18] Here $a \gg r_{0}$ and we set the range $r_{0}=0$.

[19] C. Lobo, A. Recati, S. Giorgini, and S. Stringari, Phys. Rev. Lett. 97, 200403 (2006); R. Combescot, A. Recati, C. Lobo, and F. Chevy, ibid. 98, 180402 (2007); S. Pilati and S. Giorgini, ibid. 100, 030401 (2008).

[20] M. Veillette, E. G. Moon, A. Lamacraft, L. Radzihovsky, S. Sachdev, and D. E. Sheehy, Phys. Rev. A 78, 033614 (2008); M. Veillette, D. Sheehy, and L. Radzihovsky, ibid. 75, 043614 (2007).

[21] We have obtained more stringent bounds for the imbalanced case, using the detailed structure of $\operatorname{Im} \Gamma$, but the simpler analysis described here suffices to establish a range linear in $k$ centered about $-\epsilon(\mathbf{k})$.

[22] R. Haussmann, M. Punk, and W. Zwerger, Phys. Rev. A 80, 063612 (2009).

[23] R. B. Diener, R. Sensarma, and M. Randeria, Phys. Rev. A 77, 023626 (2008); see Sec. VI and especially Eq. (34).

[24] A. Perali, P. Pieri, and G. C. Strinati, Phys. Rev. Lett. 100, 010402 (2008).

[25] Our result here corrects a factor of $\sqrt{2}$ in W. Schneider, V. Shenoy, and M. Randeria, e-print arXiv:0903.3006. [cond-mat]

[26] This large- $\omega$ tail is valid for $\epsilon_{F} \ll \omega \ll 1 / m a^{2} \leqslant 1 / m r_{0}^{2}$, where $a^{\prime}$ characterizes the final state interactions and $r_{0}$ is the range.

[27] P. Pieri, A. Perali, and G. C. Strinati, Nature Phys. 5, 736 (2009), attribute the coefficient $\Delta_{\infty}$ of their $\omega^{-3 / 2}$ tail to pairing fluctuations above $T_{c}$. Our results, however, show that this universal tail is related to the "contact" and not necessarily to pairing, either above or below $T_{c}$.

[28] R. Combescot, F. Alzetto, and X. Leyronas, Phys. Rev. A 79, 053640 (2009). 